\title{
Progress Toward Roll Processing of Solar Reflective Material
}

\author{
Russell Smilgys and Steve Wallace
} Science Applications International Corporation

$$
\text { McLean, VA }
$$

Cheryl Kennedy

National Renewable Energy Laboratory

Golden, $\mathrm{CO}$

\section{Presented at Solar Forum 2001}

NREL/PR-550-40031

$$
\text { April 23, } 2001
$$

Presented at the 2001 Solar Energy Forum: The Power to Choose held on April 21-25, 2001, in Washington, D.C. 


\section{Disclaimer and Government License}

This work has been authored by Midwest Research Institute (MRI) under Contract No. DE-AC36-99GO10337 with the U.S.

Department of Energy (the "DOE"). The United States Government (the "Government") retains and the publisher, by accepting the work for publication, acknowledges that the Government retains a non-exclusive, paid-up, irrevocable, worldwide license to publish or reproduce the published form of this work, or allow others to do so, for Government purposes.

Neither MRI, the DOE, the Government, nor any other agency thereof, nor any of their employees, makes any warranty, express or implied, or assumes any liability or responsibility for the accuracy, completeness, or usefulness of any information, apparatus, product, or process disclosed, or represents that its use would not infringe any privately owned rights. Reference herein to any specific commercial product, process, or service by trade name, trademark, manufacturer, or otherwise does not constitute or imply its endorsement, recommendation, or favoring by the Government or any agency thereof. The views and opinions of the authors and/or presenters expressed herein do not necessarily state or reflect those of MRI, the DOE, the Government, or any agency thereof. 


\section{Goal:}

Demonstrate that it is possible to cost-effectively produce high performance solar reflective material using vacuum deposition techniques.

high performance:

Specular reflectance above $90 \%$ for at least 10 years

cost effective:

$\$ 10.8$ per square meter ( $\$ 1$ per sq $\mathrm{ft}$.)

\section{Approach:}

Develop roll coating process for a first surface silver mirror with a protective alumina coating.

Focus on increasing durability and lowering production costs. 


\section{First Surface Mirror under Development to Replace Thin Glass}

\section{Top Protective Layer ( 0.5 - $\left.4 \mu \mathrm{m} \mathrm{Al}_{2} \mathrm{O}_{3}\right)$}

\section{Reflective Layer (100 nm Ag)}

\section{Metal Back Layer (optional)}

PET Substrate or (Chrome Plated Steel)

- Vacuum evaporate metal layers

- Deposit alumina coating by EB-PVD technique called ion beam assisted deposition

- Process compatible with roll coating for efficient production 


\section{Possible Application for Material: Dish/Engine System}

Mirrors arrayed on a dish concentrate sunlight onto Stirling engine

Pictured system was designed and built by SAIC (Golden, CO)

Concentrator:

- stretched-membrane faceted dish

- 90 square meters

- $1 \mathrm{~mm}$ thick silvered thin glass

- $90 \%$ reflectivity

Issues:

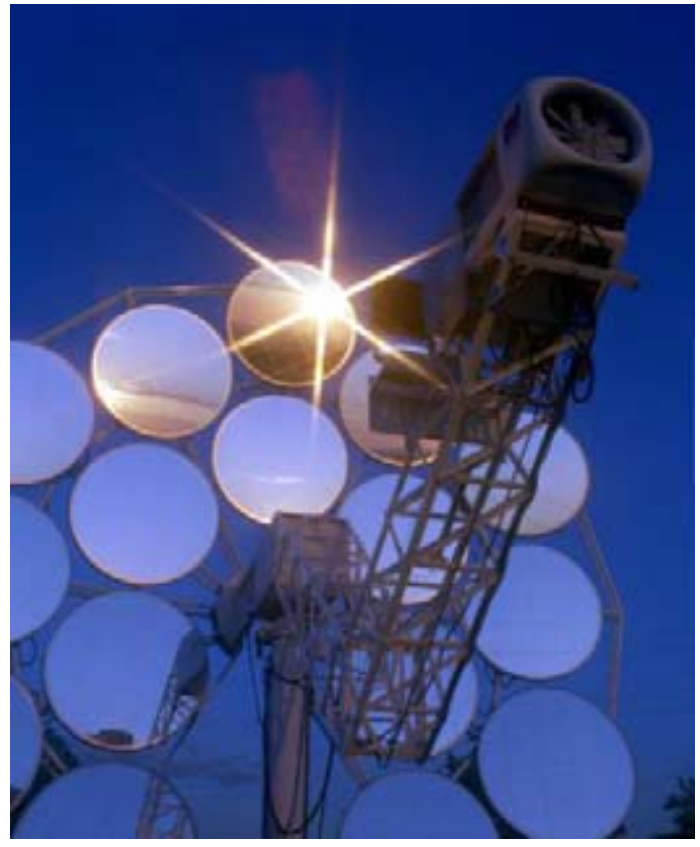

- Individual panes of glass are cut and glued to stainless steel membranevery labor intensive

-Reduction in cost of complete system possible if steel membrane could be supplied with mirror finish 


\section{Outline of Program}

1994 First sample of solar reflector material produced at Armstrong World Industries.

1995 Start of funding by NREL to make samples on PET with coating 0.5 to 5.6 microns thick using small box coater.

Prepare analysis of cost to produce material in roll coater.

1997 Build large coating system at SAIC.

1998 Increase alumina deposition rate from $1 \mathrm{~nm} / \mathrm{s}$ to $10 \mathrm{~nm} / \mathrm{s}$.

1999 Increase alumina deposition rate to $20 \mathrm{~nm} / \mathrm{s}$.

2000 Build web-handling machine and integrate into SAIC coating system.

2001 Produce solar reflector material on 12 foot long strips of chrome-plated steel web (underway). 


\section{First Test Samples Produced in Small Box}

\section{Coater}

- $66 \mathrm{~cm}$ wide box coater

- Electron beam evaporator

- Cold cathode ion source

- $8 \mathrm{~kW}$ power supply

- Quartz crystal rate monitor/ controller

- No direct cooling of substrates

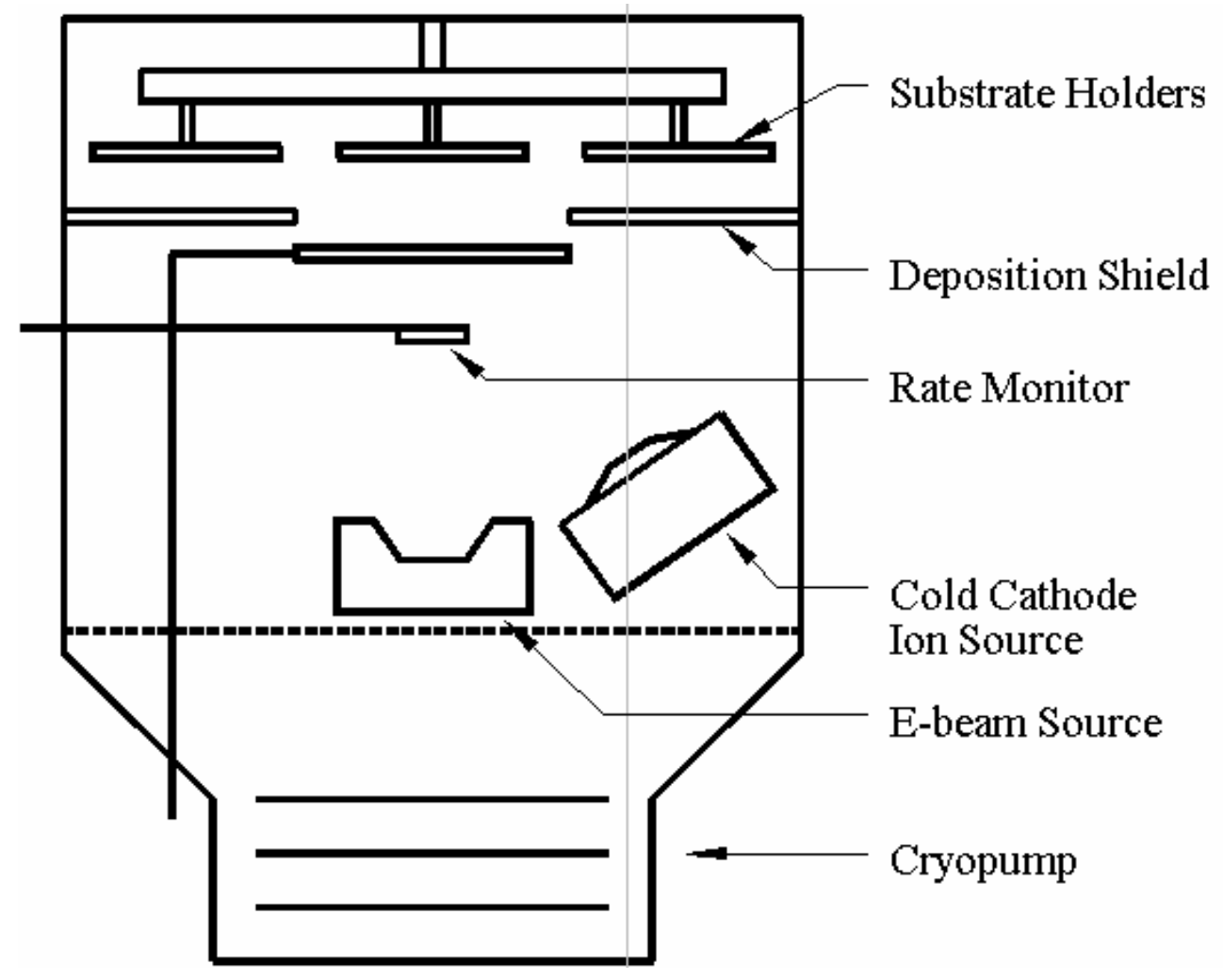

Box coater located at Armstrong World Industries, then Penn State University 


\section{Typical Deposition Procedure}

1. 6" $\times 6$ " PET sheets stretched over shim stock holder, mounted on rotating planetary holders.

2. Sputter clean PET with argon ion beam for 5 minutes

3. Evaporate copper, $50 \mathrm{~nm}$ thick $@ 1 \mathrm{~nm} / \mathrm{s}$

4. Evaporate silver, $100 \mathrm{~nm}$ thick @ $1 \mathrm{~nm} / \mathrm{s}$

5. Evaporate alumina, 0.5-4 $\mu \mathrm{m}$ thick @ $1 \mathrm{~nm} / \mathrm{s}$ 


\section{Solar Simulator Data of First Surface Mirrors with Alumina Coating (1nm/s deposition rate)}

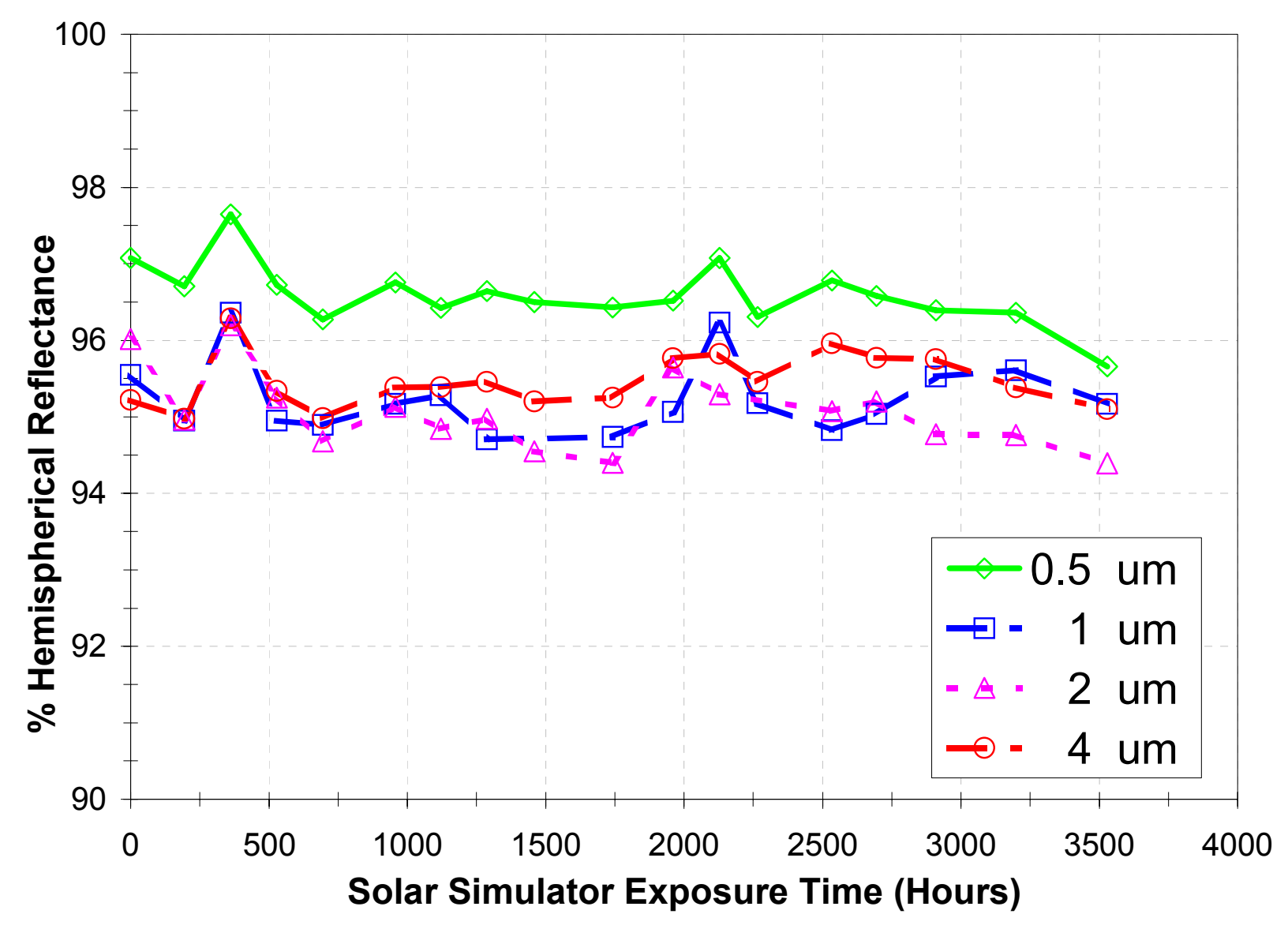

Longest durability in solar simulator of any first surface mirror 


\section{Outdoor Colorado Exposure Data of First}

\section{Surface Mirrors with Alumina Coating \\ $(1 \mathrm{~nm} / \mathrm{s}$ deposition rate)}

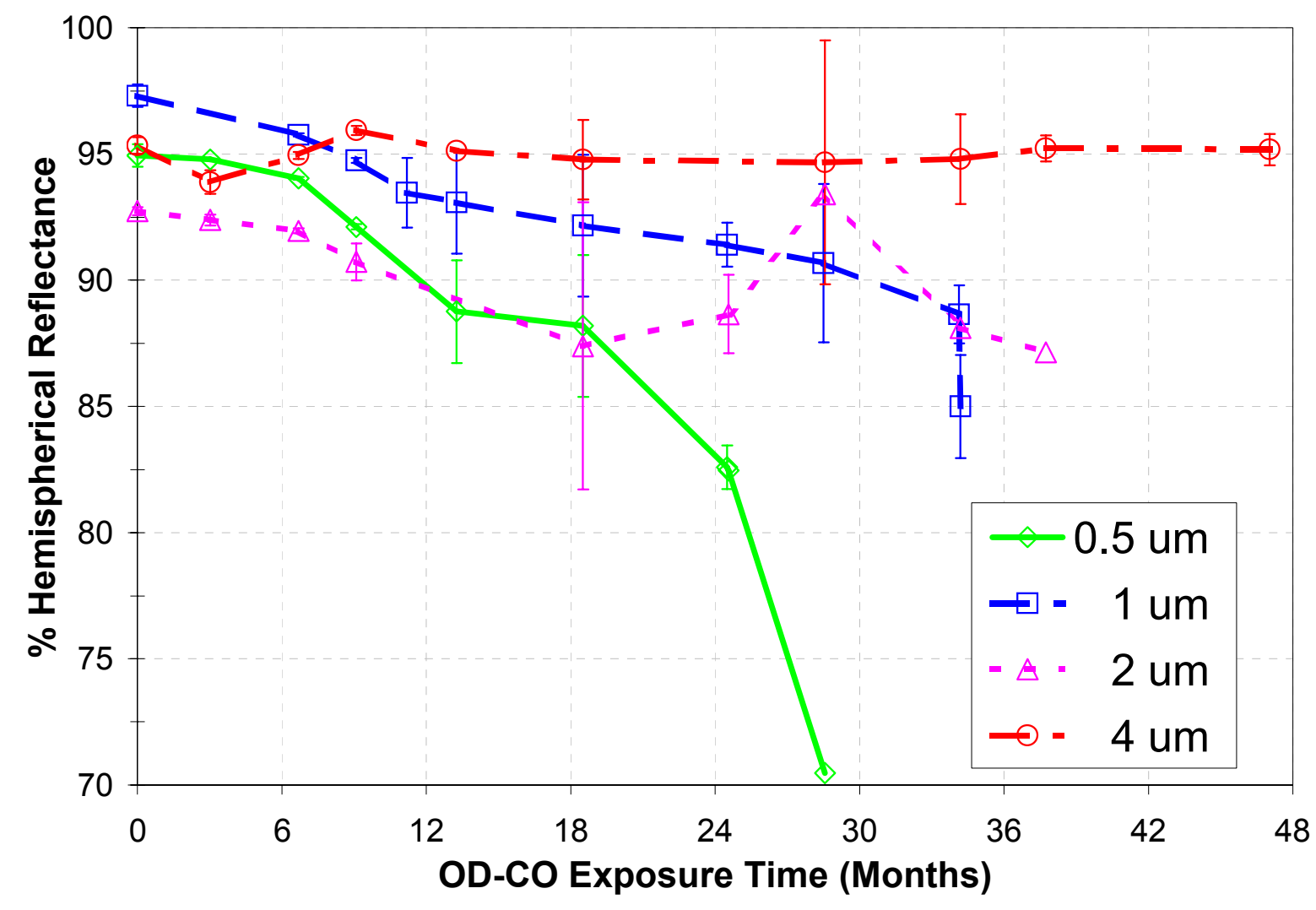

Sample with 4 micron coating lasts 4 years outdoors 


\section{Cost Model to Produce Solar Reflective Material in Roll Coater (circa 1995)}

\section{Geometry}

Web length

$2400 \mathrm{ft}$

Web width

$3.5 \mathrm{ft}$

Web diameter 12 inches

\section{Evap Process}

Capital Equipment $\$ 1 \mathrm{M}$ Web line speed $80 \mathrm{ft} / \mathrm{hr}$

Cycle time/web 30 min

Workers

$$
1 @ 100 \%
$$

\section{IBAD Process}

Capital Equipment $\$ 1.19 \mathrm{M}$

Cycle time/web

$83.4 \mathrm{hr} @ 32 \mathrm{~nm} / \mathrm{s}$ Workers

\section{Plant Operation}

300 days/yr

3 shifts/day

$8 \mathrm{hr} / \mathrm{shift}$

$15 \%$ capital recover cost

$5 \%$ capital recovery period

$\$ 35$ wage rate

$\$ 0.10$ electricity $/ \mathrm{kW}-\mathrm{hr}$

Materials Cost

Alumina

$\$ 12 / \mathrm{lb}$

Silver

$\$ 200 /$ lb wire

Copper

$\$ 2.5 / \mathrm{lb}$ wire

PET

$\$ 1.5 / \mathrm{lb}$ 


\section{Result of Cost Model to Produce Solar Reflective Material in Roll Coater}

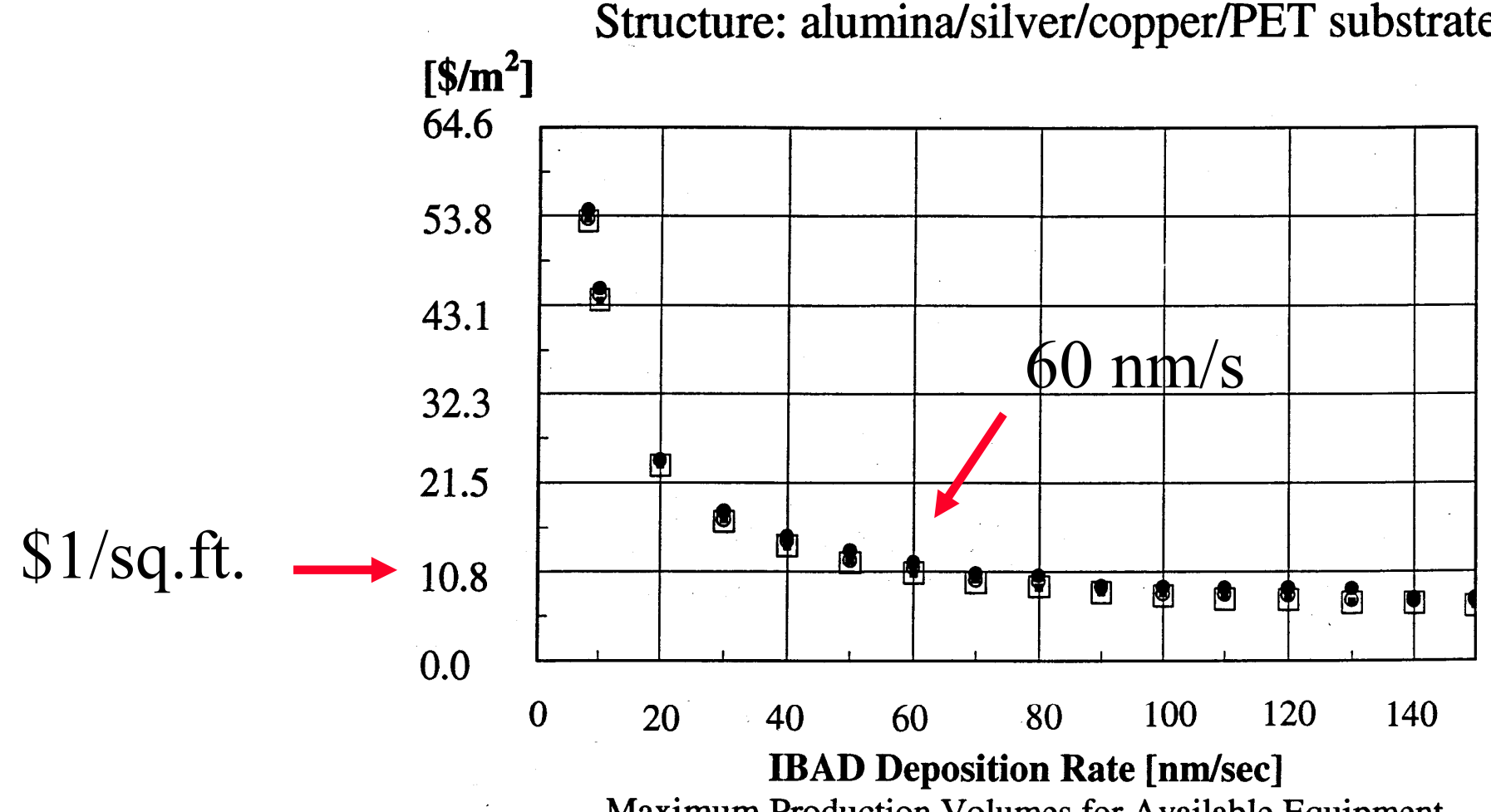

Maximum Production Volumes for Available Equipment

Film Thickness: $\mathrm{Al}_{2} \mathrm{O}_{3} 4 \mu \mathrm{m}, \mathrm{Cu} \& \mathrm{Ag} 200 \mathrm{~nm}$

When alumina coating is 4 micron thick, need $60 \mathrm{~nm} / \mathrm{s}$ rate for $\$ 1 /$ sq.ft.. (Assumes production volume one million sq. meters per year.) 


\section{Build Bigger Coating System at SAIC to Increase the Deposition Rate}

- Vacuum system walk-in sized - cylinder $7 \mathrm{ft}$ diameter, $12 \mathrm{ft}$ long

- Stokes 412 roughing pump

- three CTI-10 cryopumps

- Evaporators

- rod fed source (2" OD)

- 4 pocket source (each $60 \mathrm{cc}$ )

- $14 \mathrm{~kW}$ Temescal power supply

- Ion source

- $16 \mathrm{~cm}$ CSC gridded, high output 1.25 A@1000 V

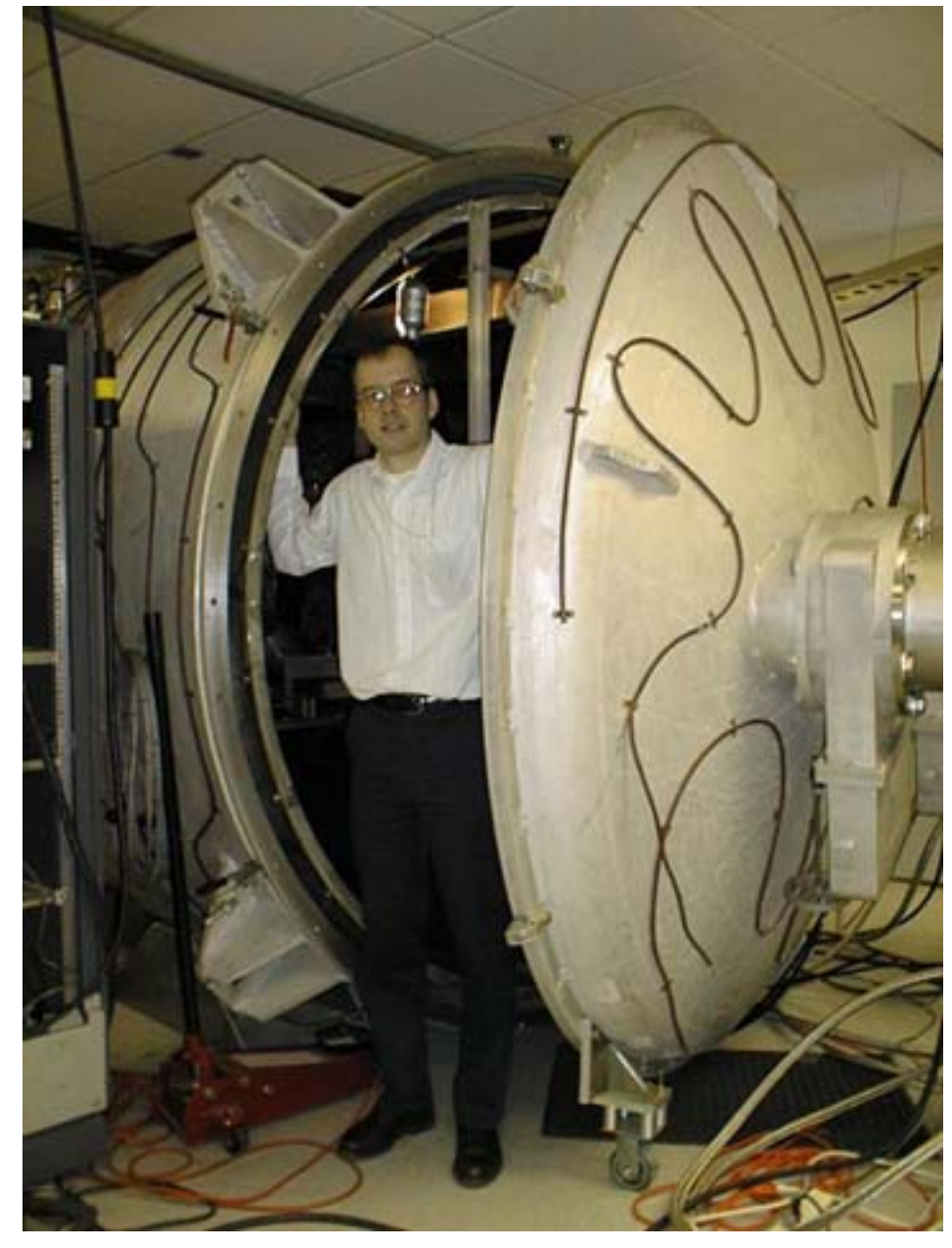




\section{Increase Alumina Deposition Rate + Sheet Size}

Cooled Al plate mounts sheets 7"x25"

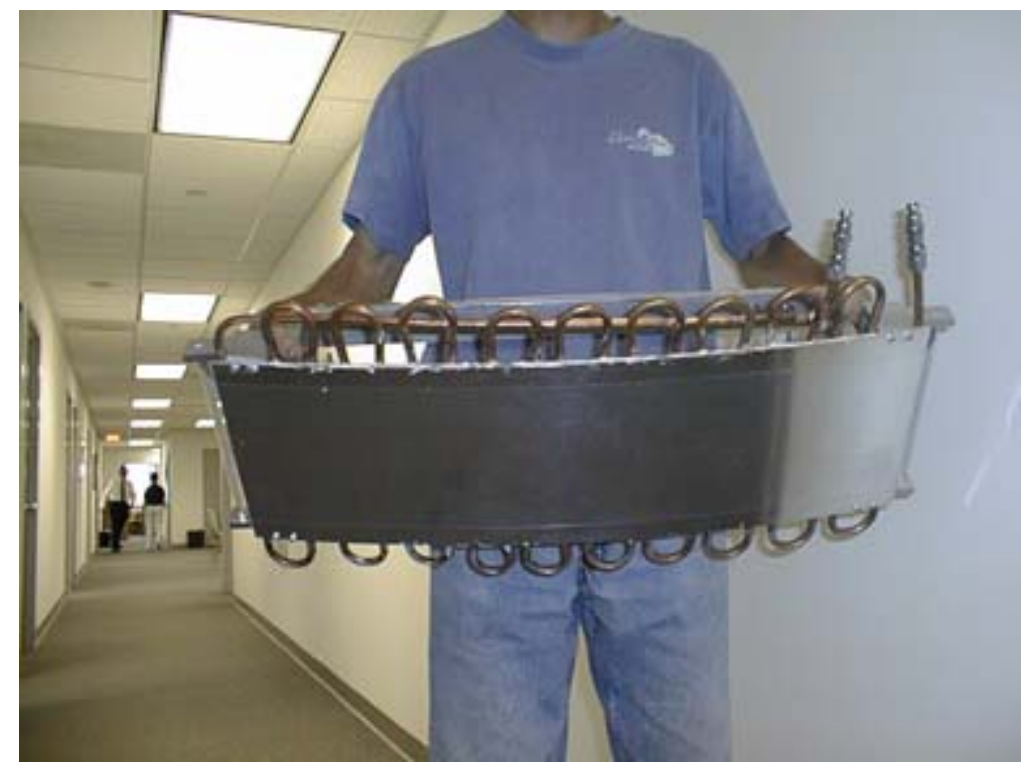

Cooled Al plate in chamber

Evaporators and ion source located at bottom of chamber

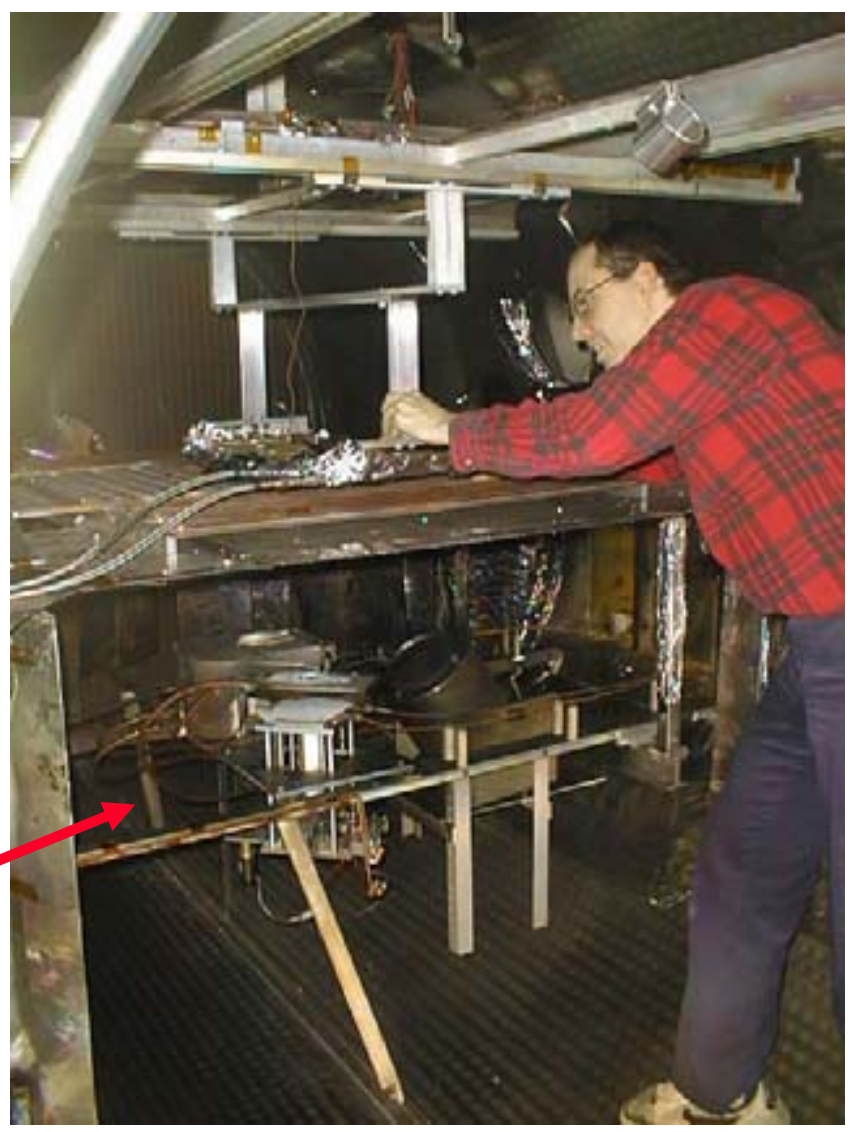




\section{WOM Data of First Surface Mirrors with Alumina Coating (higher deposition rate)}

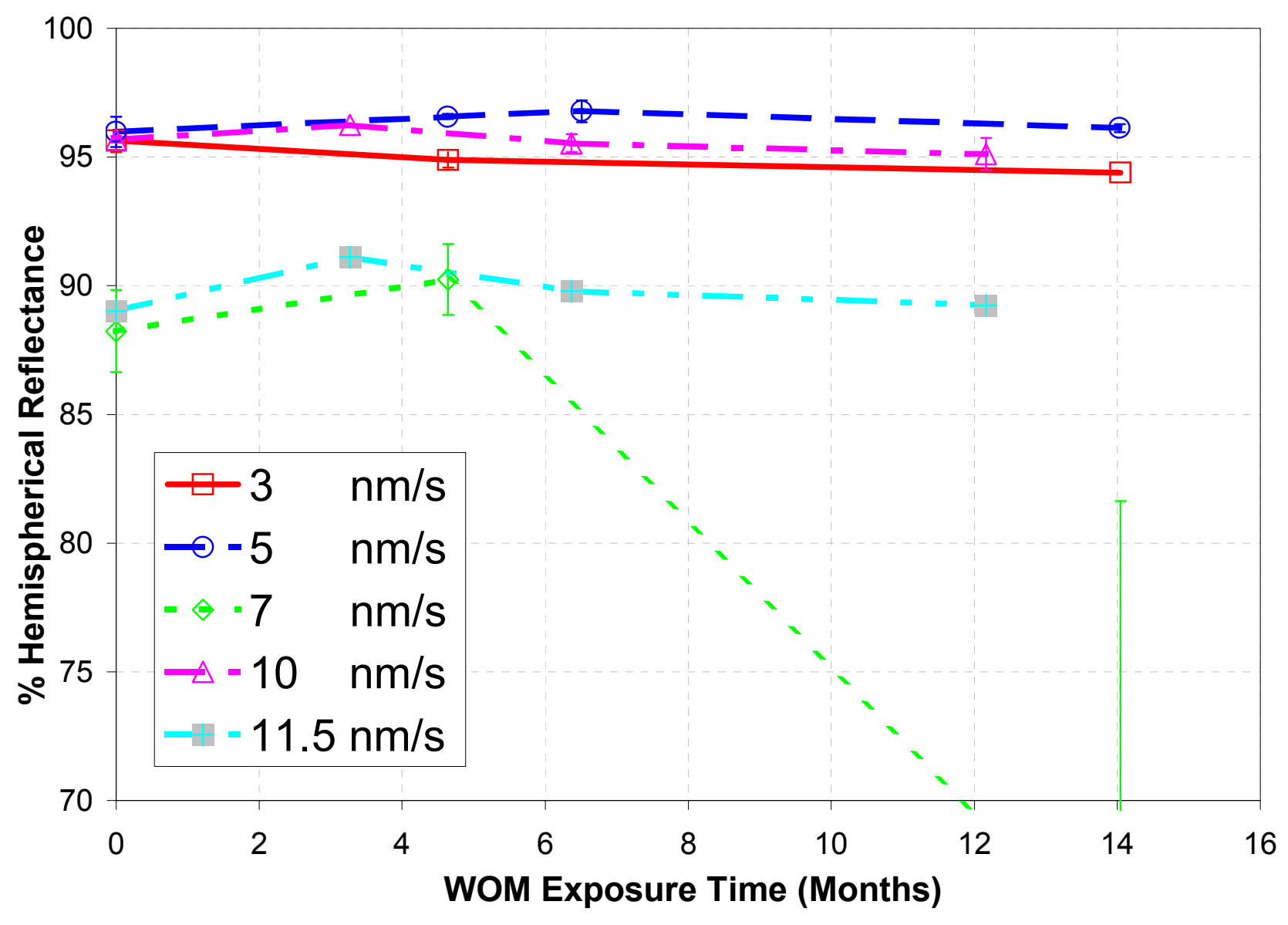

Samples with 10x increase in deposition rate still durable. 


\section{Roll Coater Design}

\section{Specifications}

Web width $30.5 \mathrm{~cm}$

Web speed $1-89 \mathrm{~cm} / \mathrm{min}$ Web tension 1-189 N

Reel ID $30.5 \mathrm{~cm}$

Drum temperature $>77 \mathrm{~K}$

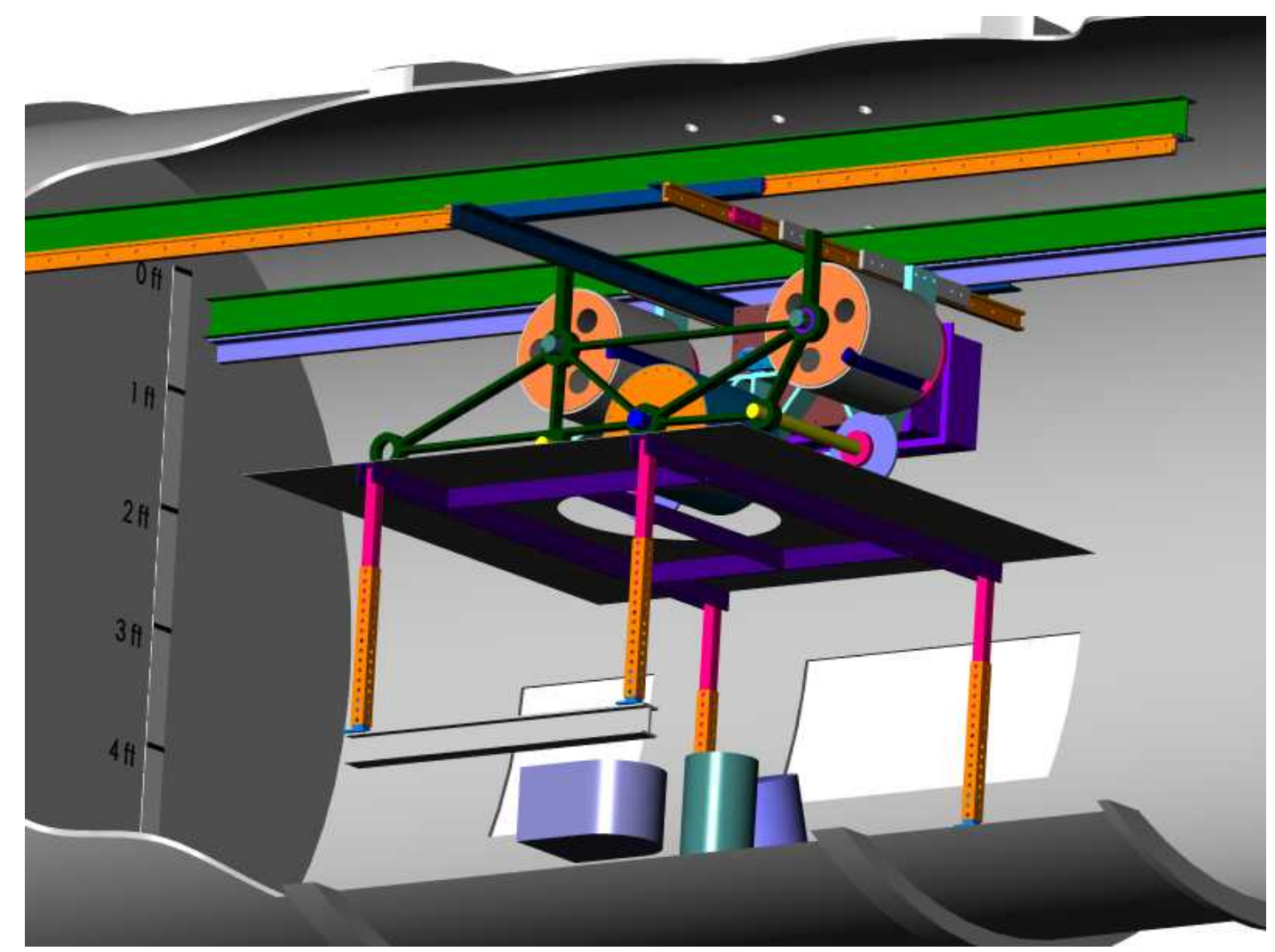

Intended web is chrome-plated steel strip, 12 " wide, 8 mil thick 


\section{Roll Coater at SAIC (McLean, VA)}

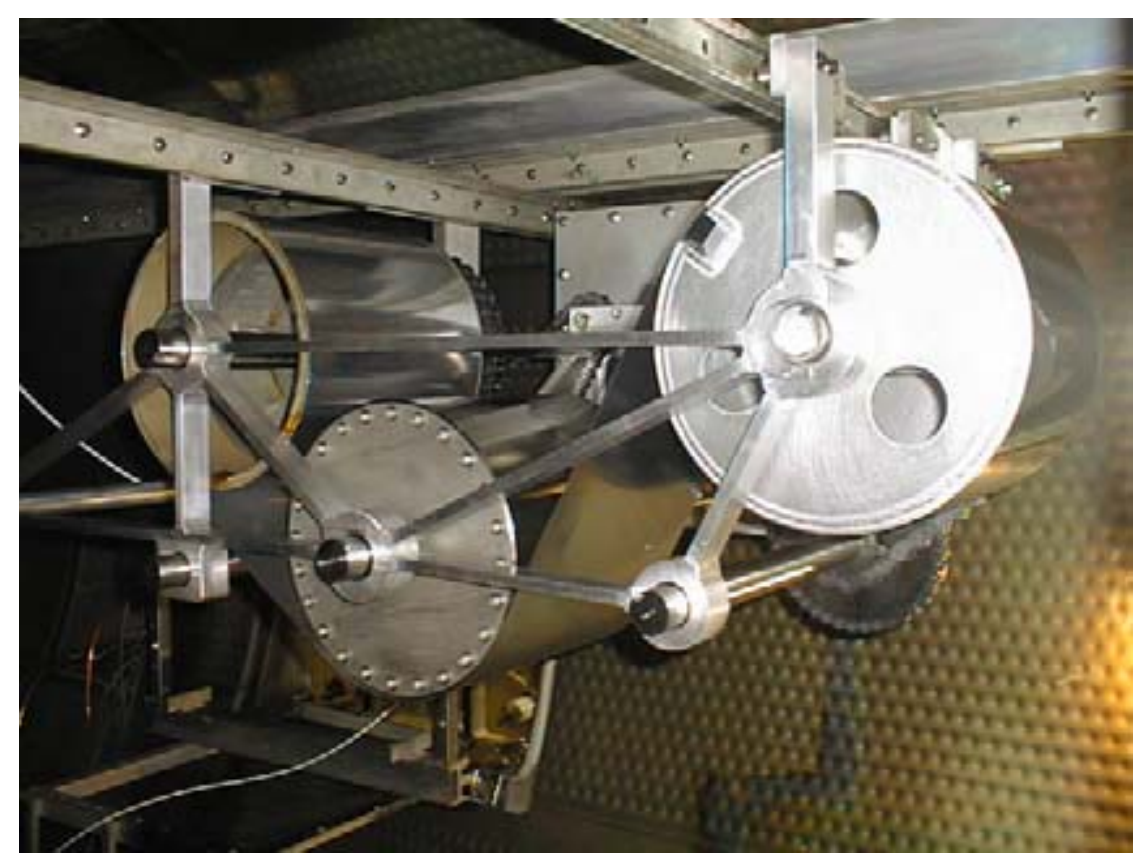

Front view showing steel web advanced over $12 "$ drum

Rear view showing chain drive
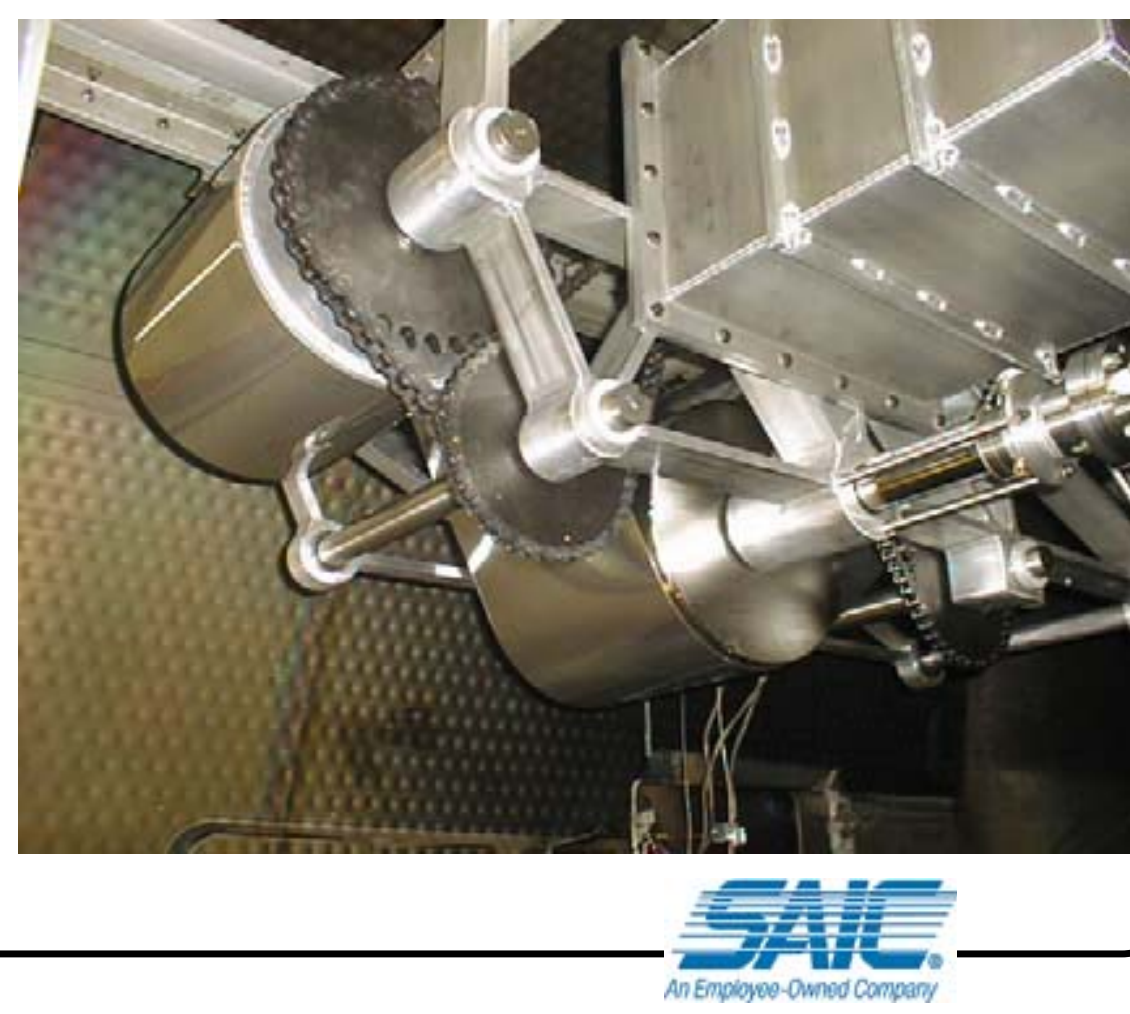


\section{Roll Coater at SAIC (McLean, VA)}

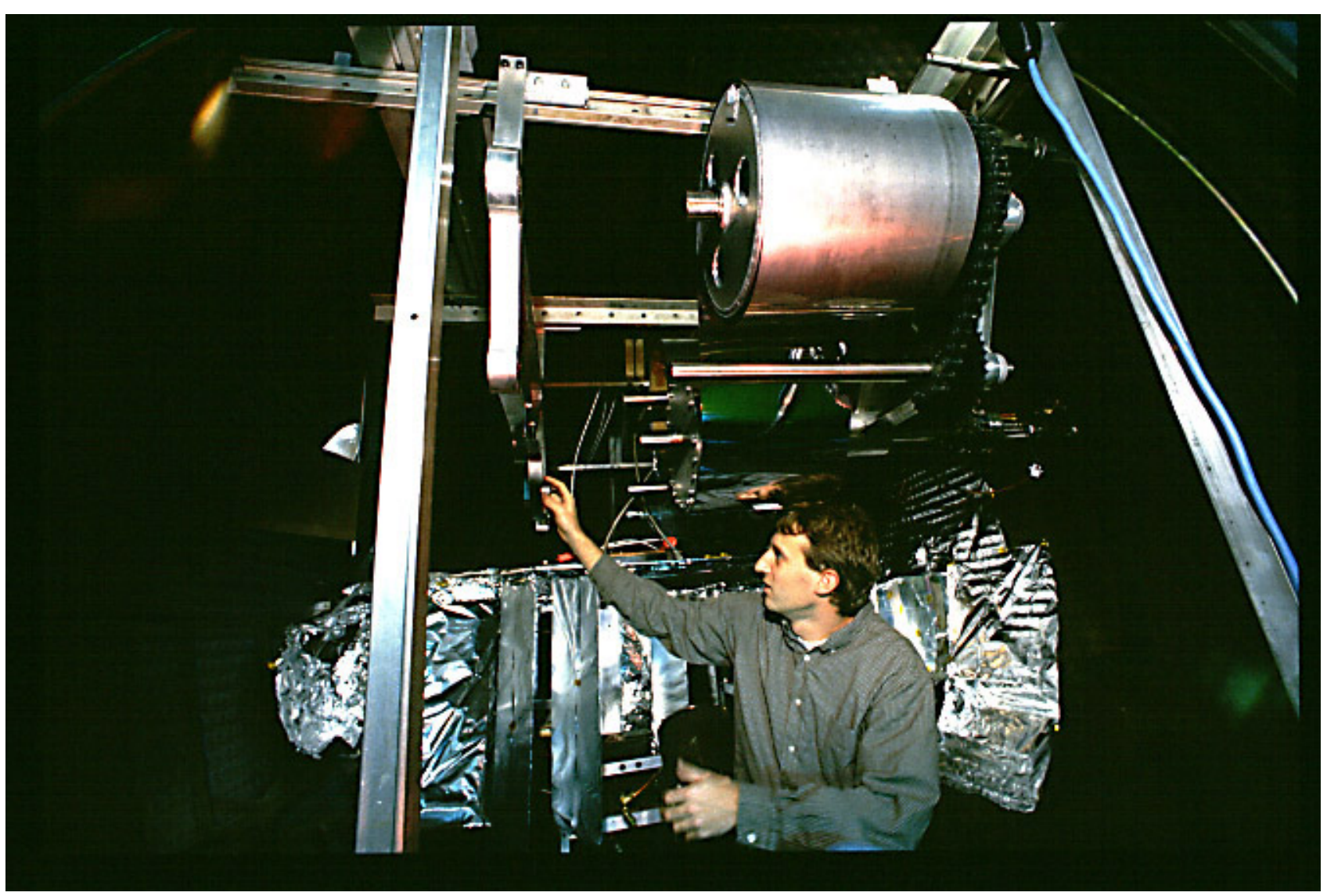




\section{Development Path to Commercial Application of Solar Mirror}

Current Work:

- Transition coating process from PET to chrome-plated steel web (12" wide).

- Produce solar reflective material on 12' long strips of chrome-plated steel.

Future Work:

- Produce 280 lineal feet of solar material for field trials.

- Increase the alumina deposition rate beyond $20 \mathrm{~nm} / \mathrm{s}$. 


\title{
Acknowledgments
}

\author{
Northeastern University \\ Dr. Jackie Isaacs
}

Armstrong World Industries:

Dr. J. S. Ross

Penn State University:

Prof. Singh

Tom Medill

Dale Donner 